\title{
Optical communication system for military applications
}

\begin{abstract}
This article deals with the study of intercommunication systems used by satellites that use optical link and its ultimate goal is the construction of an optical receiver in a printed board. The optical receiver was represented by a diagram of blocks. The choice of components was made based on the advantages and disadvantages which have, each one being explained and analyzed. Taking into account the different options that can be carried out for each block, was chosen as the most suitable and beneficial to the entire set of components that the recipient will have. Throughout this article was made an introduction and explanation of each stage of the recipient, as well as of its components. To complement, tests were performed practical on each block, and sometimes the main component in specific to that same block. It is concluded that the modeling and preparation of optical receiver for low frequency, is able to meet all the objectives set forth, may equate this solution to a receiver capable of operating in a satellite in orbit of space.
\end{abstract}

Volume 5 Issue 2 - 2019

\author{
Carlos Eduardo Fatela,' João Paulo N \\ Torres, ${ }^{2}$ António Baptista, ${ }^{3}$ Maria João \\ Martins' \\ 'Academia Militar, Portugal \\ Instituto de Telecomunicações, Portugal \\ ${ }^{3}$ Instituto Superior Técnico da Universidade de Lisboa, Portugal
}

Correspondence: João Paulo N Torres, Instituto de Telecomunicações, Instituto Superior Técnico da Universidade de Lisboa, Lisboa, Portugal, Email joaotorres@ist.utl.pt

Received: June 09, 2017| Published: April 08, 2019

Keywords: communication systems, lasers, optical communications, radiofrequency, satellites

\section{Introduction}

Artificial satellites play an increasingly important role in people's lives and as such, there exists a set of interests in its technological development in order to use all the opportunities and take advantage of its benefits. You must have a system for efficient communication between the satellites for which the sending and receiving of data is done quickly and without loss of information. The failure of the temporal goal in the delivery of information may make it useless and may even lead to wrong decisions. The mobile communications, movements of the fleet, terrorism, among other things, require monitoring and ongoing maintenance. There is therefore a big bet in satellites according to their usefulness regarding the sustainability of the resources that they provide. Provide and maintain some services such as television, internet or even provide the geographic location of the user, with the use of GPS (Globlal Positioning System). ${ }^{1,2}$ With regard to optical networking, its evolution can be divided into two periods: at the end of the 1980 s, when the nets were used only to transmit data i.e. the perspective was only used for transmission of information. The second period where they began to use the perspective to transmit and perform the functions of routing and switching (routing and forwarding), allowing you to obtain economic benefits since there would be no need to use considerable amounts of electronic components. The use of optics in the components has resulted in many benefits such as: the increment of dynamics in the network, algorithms to find, select, shortest paths in communications, capacity to prevent and resolve transmission faults, among others. ${ }^{3}$

There are several issues that after they have been tested, justify the use of communications optic compared to the use of communications by microwaves in data transmission. The advantages when compared to the older communications systems are the following ${ }^{4-8}$ :

a. The absorption and dispersion are effects which, in free space, can be overlooked

b. Increased bandwidth and the use of bands narrower per channel preventing counter-spying

c. transmission speeds, with debts torque levels

d. Low transmission power due to the characteristics of the optical receiver and its mechanisms

e. Smaller physical dimensions

f. More economical for the payment of fees and regulatory constraints

It is in this sense that the interest in the study of optical communications, more specifically, the analysis and on the receiver optics a communications system. Are analyzed and described the blocks that are with the use of Software, and subsequently, practice in the laboratory.

\section{Optical communication system}

\section{Satellites}

The term satellite used today is derived from the Latin word satelles or satellites whose meaning is a body that gravitates around a star of earth role, as for example, the planet Earth.

Due to the high number of satellites already constructed by man it is difficult to group them according to various categories. Thus, artificial satellites, which are the ones that require particular attention in this work, will be divided three groups':

a. Military satellites: they had defined as goals are connection points for telecommunications, observation points on the enemies and consequently alerts, served to navigation and recognition in areas unknown, among others.

b. Scientific Satellites: these types of satellites include meteorological satellites, satellites and data collection. Have the function to indicate the climate so as to avoid, allow you to 
better understand the space that surrounds us and try to justify the physical phenomena, chemical or biological, which arise in the Earth.

c. Satellite navigation: the 24 satellites for navigation form a constellation that is approximately $20,000 \mathrm{~km}$ altitude thus forming the support for the GPS system.

d. Communications Satellites: these satellites are those that serve as the basis for the sending and reception of data, allowing for the dissemination of information to any location in the world. With respect to the orbits, can be grouped the satellites according to four different orbits"

e. LEO (Low Earth Orbit): The satellites with this type of orbit were used in communications that are not maintained directly linked for a long time. Had the aim of act as sensors in scientific or military.

f. MEO (Medium Earth Orbit): This type of orbit can be divided into two groups, orbit semi-synchronous and the Molniya orbit. The orbit semi-synchronous regulator to $26,560 \mathrm{~km}$ of land surface and has little eccentricity. ${ }^{1}$ The GPS works with satellites that perform this type of orbit. The orbit of Molniya, invented by the Russians, works effectively for observations of high latitudes.

g. HEO (High Earth Orbit): The satellites with this orbit are typically used for communication purposes. Are placed between $35800 \mathrm{~km}$ and $360000 \mathrm{~km}$ altitude and accompany the rotation of the earth at the same speed.

These orbs can be assigned to geosynchronous orbit. If the orbit of the satellite is around the equator, the correct name is geostationary orbit.

Regarding the classification of the satellites with respect to the use of the frequency band, the following table summarizes the existing bands and their respective frequency range (Table 1):

Table I Bands of communication and their frequency ranges

\begin{tabular}{ll}
\hline Band & Frequency $[\mathrm{GHz}]$ \\
\hline $\mathrm{P}$ & 0.2 to 0.4 \\
$\mathrm{~L}$ & 1.53 to 2.7 \\
$\mathrm{~S}$ & 2.7 to 3.5 \\
$\mathrm{C}$ & 3.7 to 4.2 \\
$\mathrm{X}$ & 5.725 to 6.425 \\
$\mathrm{KuI}$ (PSS) & 7.9 to 8.4 \\
Ku2 (DBS) & 10.7 to 11.75 \\
Ku3 (Telecom) & 11.75 to 12.5 \\
Ka & 12.5 to 12.75 \\
$\mathrm{~K}$ & 17.7 to 21.2 \\
\hline
\end{tabular}

\footnotetext{
${ }^{1}$ The eccentricity of an orbit indicates the difference that the orbit is compared to a perfect circle. A circular orbit has an eccentricity 0 , while an eccentric orbit has a value of almost 0 . A satellite is an eccentric orbit that rotates around a point and not the center of the Earth. ${ }^{10}$
}

\section{Optical system}

Communication system optics has in general three characteristics that quantify: the transmission power, the loss of the spread and the sensitivity of the recipient (amount of power required that the recipient needs for your components work properly). You can also split a communication system in the following manner: the issuing module, the means or channel of spreading, and the receiver module. The part of the recipient is constituted mainly by a photodetector, by electronic components for amplification, by a differentiator, by circuit for recovery of signal and alarm, for a demodulator and a decoder (Figure 1). ${ }^{5,10}$

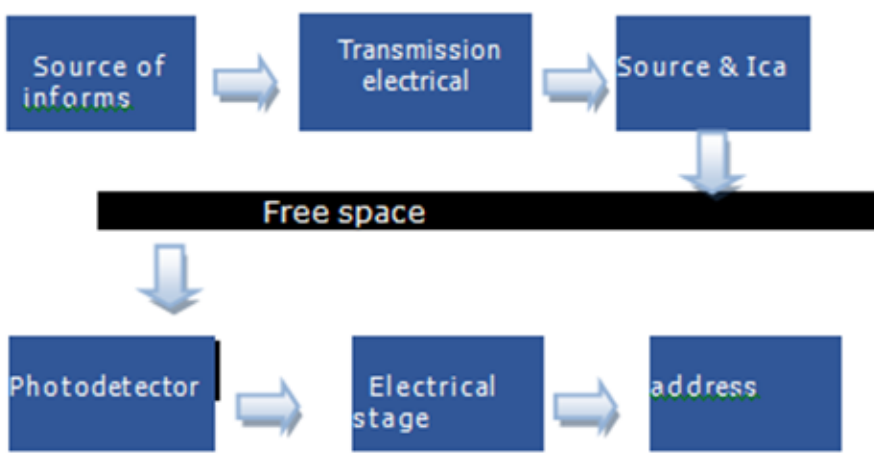

Figure I Optical communication system.

The part of the canal of 24 can also be divided into two parts: the detection of the signal and the reception of the signal. The part of the face detection consists of a component whose physical area decreases with the increase of the bandwidth used. The advantage of having physical dimensions minors is the decrease of black noise. As the lasers used in these situations operate in a regime of natural count of photons (the photons are counted one by one) there is an increase of $8 \mathrm{~dB}$ to $10 \mathrm{~dB}$ on connection SNR. As for the reception of the signal, there are some limitations to take into account, as for example, the efficiency of the detector, the black noise, the time of the jitter and the limitations of electronic components or optical. ${ }^{10}$

\section{Modulation types}

You can describe the modulation as the process in which the data to be transmitted in a communication are added to electromagnetic waves. Through the process of modulation is accomplished by the passage of an analog signal to a digital signal. This process may vary, the intensity, frequency, the wave length, among other characteristic parameters of the signal by deforming the characteristics of the signal (amplitude, frequency or phase). These characteristics will change depending on the signal modulator. The methods of modulation can be divided into three types: the modulation in amplitude; modulation frequency; and the modulation on stage. In the modulation in amplitude is varying the amplitude of the waves of signal in operation. Amplitude encodes for example the bit " 1 " and the other amplitude encodes the bit " 0 ".

In the modulation in frequency changes the frequency or number of cycles per second $(\mathrm{Hz} / \mathrm{s})$ and in this way, a frequency is used to encode a signal, a bit, and another frequency will be used to encode the other bit. In the modulation by phase, as its name indicates, is the stage of the waves, reversing their direction. Variations can be multiple and every variation of ph can be encoded multiple bits. Even on the 
modulation in frequency, this can be directly modulating or indirect modulation. The direct modulation is to modulate the amplitude of the optical beam however, has the effect of chirp (overload setting, i.e., the maximum power that the recipient can accept the entry, usually up to $0 \mathrm{dBm}$ ). This fact strongly degrades system performance due to processes in the laser cavity. In modulating indirectly the effect of chirp is eliminated but the complexity and the loss of the receiver increases. As the formats of modulation that can fit in communications.

The modulation On-Off Keying (OOK) is one of the formats of modulation more simple that exists and is considered as a case of modulation in amplitude: each bit is placed in a time interval (time slot) and each such interval corresponds to a symbol, i.e. is a technique simple binary. To be sent the bit " 1 " is issued a laser beam, in the absence of this beam is considered that if you want to send the bit " 0 ". The receptor, each period of time, decide what bit received. The waiting time is inversely proportional to the rate of connection which implies a bandwidth of operation with a value equal to the value of the rhythm. The pulses used in this case will have to be necessarily unipolar, the type NRZ (Non Return to Zero), in which the impulse has the same duration as the period of the bit, or the type RZ (Return to Zero) in which the impulse will have a shorter duration in relation to the period of the bit. The impulses used are the impulses NRZ because they require a lower bandwidth on the part of the recipient and are still simpler. To determine what the bit received the recipient, to make the demodulation of the signal, compares the amplitude of the received signal with a threshold value and register if you received the bit zero or one bit. This Modulation allows you to have a value of BER quite acceptable for low torque rhythms (Figure 2). ${ }^{11-13}$

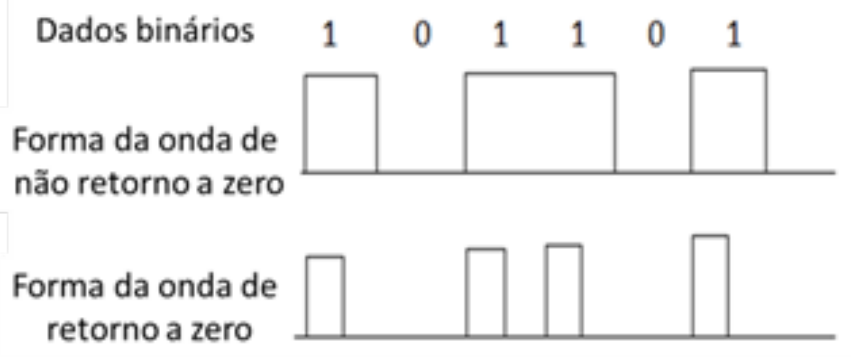

Figure 2 Modulation OOK.

The expressions in this modulation are the following:

$$
T_{\text {slot }}=\frac{1}{D_{b}}
$$

$T_{\text {slot }}$ is the duration of a time range and $D_{b}$ is the rate.

$$
B_{w}=\frac{M D_{b}}{k}
$$

The bandwidth supply $B_{w}$ depends on the modulation used, $k$ is the number of bits and the degree of modulation.

It is important to note that the power levels maximum, minimum and average of a signal laser when it sends the signals. Figure 3 is a schematic figure simple to understand the powers of signals used in this modulation.

An aspect since it is important that, in cases ideals, the relative potency to bit zero is zero but, in actual cases, this does not happen and the expression $P_{\max }=2 P_{\operatorname{med}}$ is not valid.

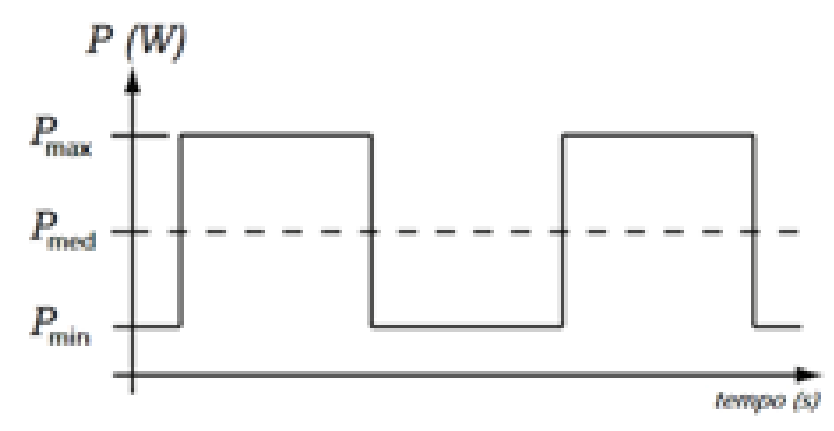

Figure 3 Power Levels.

Through the ratio of extinction (reason that quantifies the optical power required for the zero logical if extinguished) can relate to maximum power and minimum power allowing their expressions. The expression (4.3) indicates the ratio of extinction:

$$
r=\frac{P_{\min }}{P_{\max }}
$$

Both powers, maximum and minimum pressure, can be obtained through the ratio of extinction and average power:

$$
\begin{aligned}
& P_{\max }=\frac{2 P_{\text {med }}}{1+r} \\
& P_{\max }=\frac{2 P_{\text {med }}}{1+r} \cdot r
\end{aligned}
$$

In modulating OOK, the average power is defined as follows:

$$
P_{\text {med }}=\frac{P_{\max }}{2} 1+r
$$

Very briefly, this modulation technique is simple and inexpensive to implement but since it is a technique of modulation in amplitude, suffers a negative effect of attenuation of the propagating material being ineffective in environments with high losses such as the Earth's atmosphere.

The modulation Pulse Position Modulation (PPM) uses symbols with a fixed size in the scale of the time to transmit the information. These symbols are divided into $\mathrm{M}$ temporal intervals. Are the numbers of intervals of time that will determine the size of the symbols (Figure 4).

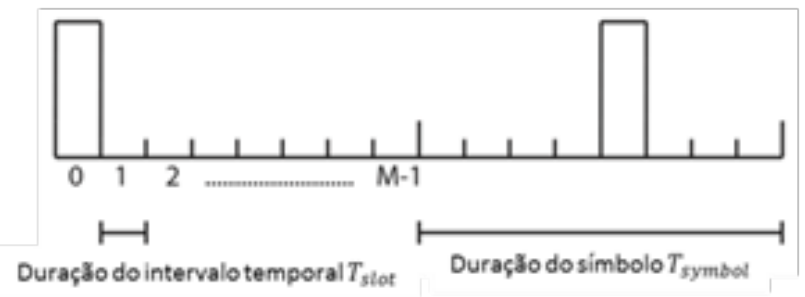

Figure 4 Signal PPM.

Figure 4 illustrates a signal PPM as well as what is considered with the symbol and the time interval of time. The expressions of the relations of the characteristics of the systems are as follows:

$$
M=k^{2}
$$

The number of bits is represented by $\mathrm{k}$ and $\mathrm{m}$ is the order of the modulation used, as has already been mentioned previously. 


$$
T_{\text {symb }}=\frac{k}{D_{b}}
$$

$T_{\text {symb }}$ is the duration of the symbol and depends on the bitrate $D_{b}$.

$$
T_{\text {slot }}=\frac{k}{M D_{b}}
$$

$T_{\text {slot }}$ is the duration of a temporal interval.

$$
B_{w}=\frac{M D_{b}}{k}
$$

The bandwidth is dependent on the modulation used.

$$
P_{\text {med }}=\frac{P_{\max }}{M}(1+r .(M-1))
$$

This modulation becomes more efficient when greater the value of modulation because the number of bits sent per symbol increases, so it is more efficient than the modulation OOK requiring only that the $\mathrm{k}$ value is greater than $2(\mathrm{k}>2)$. Yet these techniques is more complex and require that the receiver is synchronized with the beginning of each symbol to decide correctly what the bit received, so that the next stage of decoding, is done effectively. ${ }^{13}$

The technique Binary Phase Shift keying (BPSK) is a technique of digital modulation to transmit data through the modulation of the phase of a signal. They are used two stages in the signals, separated by $180^{\circ}$, and the phase is reversed in the transition from one bit to another. Figure 5 is an example of this technique of modulation.

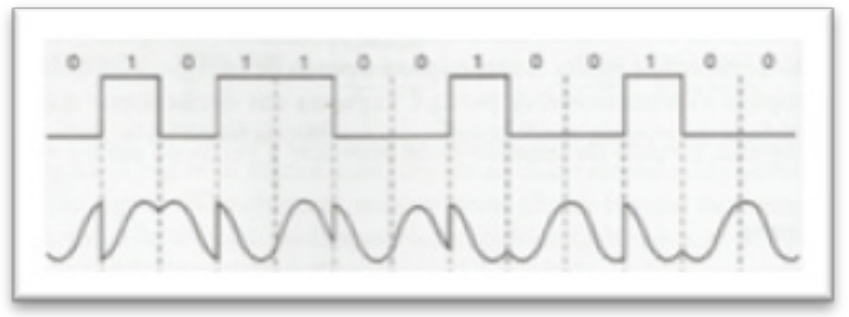

Figure 5 Modulation BPSK. ${ }^{13}$

This modulation is the most robust of all variations that arise from the PSK, since it takes as a reference the highest level of noise or distortion, so that the modulator, through this value and by comparison, come to a correct decision. The value of BER in modulating BPSK is given by the expression (2.12) where erfc is the error function complement and SNR (Signal Noise Ratio) is the signal to noise ratio of the receiver. The table of error function can be viewed in.

$$
B E R=\frac{1}{2} \operatorname{erfc}(\sqrt{S N R})
$$

The BPSK modulation can be regarded as a modeling disadvantage since only sends one bit per symbol. ${ }^{12}$

Finally, the modulation Quadrature Phase Shift Keying (QPSK) This type of modulation, derives from the PSK modulation and uses parameters of ph and squaring of the carrier wave ma model the data signal. Uses four stages by which the encoding of data is done in symbols of two bits. It is possible to carry twice the amount of data transmission using the same bandwidth when compared with the technique BPSK. The following figure is a simple example of encoding QPSK, the carrier may take four different symbols, each symbol with two bits, dibit, corresponding to $45^{\circ}, 135^{\circ}, 225^{\circ}$ and $315^{\circ}$ (Figure 6).

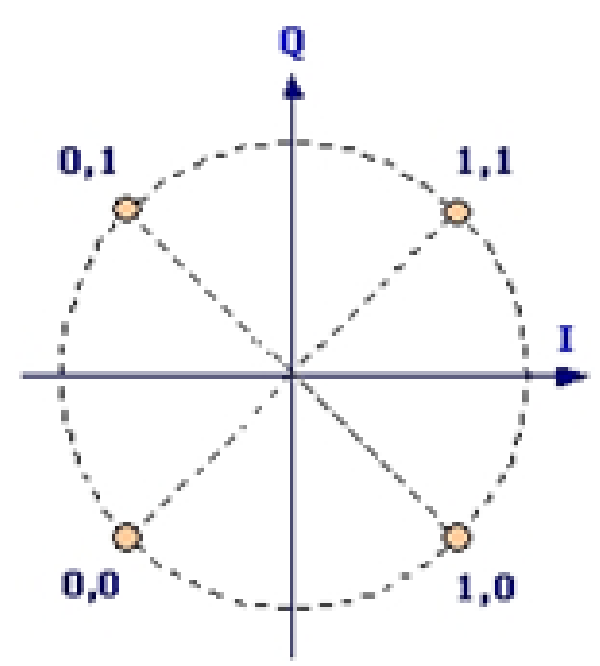

Figure 6 Phase and squase diagram. ${ }^{13}$

You can play the same role as the previous technique needing only half the bandwidth that would be required. The equation of the percentage of errors binaries for the modulation QPSK is given by the following expression in which the parameters have already been explained earlier: ${ }^{14}$

$$
B E R=\operatorname{erfc}(\sqrt{S N R})
$$

\section{Pointing tracing and acquisition system}

The system of pointing, acquire and screening, is to seek and do the screening of laser beam from the emitter as well as directing its terminals in the direction in which the sender sends its beam to which the communication between both is successful. For the purpose of this system are three operations, the phase of acquisition, the phase of screening and the phase-pointing. ${ }^{8}$

Each operation is performed by one of the 3 systems. Each system is controlled by a set of electronic components different: the electronics of acquisition and processing, the electronics of coarse pointers (coarse pointing) and the electronics of pointers (fine pointing). These systems are essential to establish good communication between satellites located at long distances. To achieve the tasks PAT there are two possibilities that can be adopted: a device that electromechanical applies a binary force the appliance optical, or a mirror MEMS (Micro Electro Mechanical System) is used to forward the optical signal in the direction desired. This last option is more susceptible to radiation and therefore the best solution to take is the electromechanical device, since it is the most robust. ${ }^{8}$

\section{Optical receiver}

\section{Operation and characteristics of the optical receiver}

Fundamentals: A simplified example of a block diagram of the optical receiver is outlined in Figure 7. This diagram shows what features, that components and systems of the receiver optics need to play, for the proper functioning of the optical receiver. ${ }^{3,15}$ 


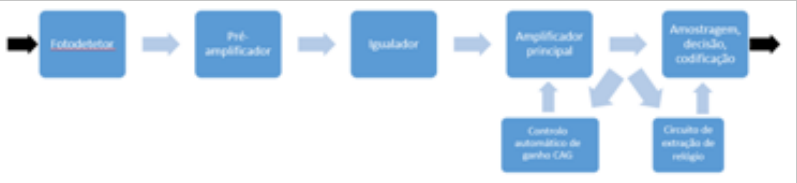

Figure 7 Example of the constitution of the optical receiver. ${ }^{10}$

With respect to the operation of the optical receiver, the first part of the receiver is a photodiode that can be of type PIN or ODA. The photodiode will produce a given electrical current. This current passes through an amplifier that is in the designated front end. After the signal is amplified, it is subject to a filter low pass. The use of a Bessel filter reduces noise, eliminates frequencies outside the bandwidth of the receiver and reduces distortion, avoiding interference inters symbolic (interference caused by the response at the time of the channel, with a modification to the information symbol transmitted). Finally, with a circuit of recovery and a system of decision, the receiver can determine which of the received signals (inputs) correspond to the bits " 0 " and " 1 ". ${ }^{16,17}$ It is within this last part, which is the parameter of the error rate tightening, BER. A system of sending information will have an optical laser, an accountant of photons and a circuit for a decision. The quantum counter never reaches the desired efficiency of $100 \%$ compared to the losses and the noises, all introduced by the mechanisms involved, as has already been mentioned previously. This device will count almost in its entirety all photons received by the laser. The Decision, on the basis of the photons recorded estimates the bits that were actually received. This estimate is made by means of a statistical model, according to the Poisson distribution. ${ }^{2}$ For example, to achieve a BER of $10^{-9}$ you will need 20 photons per bit (bit "0" or "1") or an average of 10 photons per bit in the transmission. This limit of optical reception is assigned the name of quantum limit. ${ }^{2}$

The BER is inversely proportional to the quality factor $(\mathrm{Q})$, i.e., the probability of system error increase, the BER also increases. The quality factor is the measure that quantifies the signal quality and the higher this value, the better the system. When the standard deviation of the bit " 1 " is equal to the bit " 0 ", it can be said that the BER is a function of SNR. 5,16

\section{Requirements of optical}

The receiver optics must have certain requirements:

a. Physical compatibility with the satellites;

b. High Gain and low noise on the received signal; c. Response time too low when in operation with the sender optical;

d. Low costs and long durability; â

e. High responsivity ${ }^{\circledR}$.

The responsiveness of the system is described in the expression (5.1):

$$
R=\frac{I_{p}}{P_{i}}=\frac{\eta q}{h v}=\frac{\eta \lambda}{1.24}
$$

\section{Quantum efficiency}

The quantum efficiency is the ratio between the number of pairs electrons-hole generated by each photon incident. Some of the photons incidents do not create a pair electron-hole. The responsiveness (R) quantifies this effect and increases the lower the frequency used. ${ }^{2,16}$ A photodetector of type PIN, the quantum efficiency is lower than 1. The quantum efficiency of a photodiode PIN is given by: considering the optical power incident as $P_{o}$, a photodetector PIN type with a particular quantum efficiency and a certain responsiveness, and existence of a chain on photodetector $I_{p}$, then:

$$
\eta=\frac{\text { taxa de geração de pares electrão }- \text { buraco }}{\text { taxa de fotões incidentes }}=\frac{I_{p} / q}{P_{o} / h v}
$$

\section{The noise and its components}

The noise may be coming from the various components or activities. For example, it may be the source, the interaction of multiple modes are consistent, the photodetector or pre amp, the distortion caused by the non-linearity's or even of reflections that occur on the couplers. There are three main sources of noise from spontaneous fluctuations in optical communications. Figure 8 allows the framing of a simplified form some noises and their origin ${ }^{2}$ (Figure 8).

\section{Types of detection}

The receiver of direct detection presents a simple constitution, and does not depend on the stage and the frequency of the signal. Is used, in general in all the receivers. Specifically, the receiver collects and identifies the photons received, i.e., it is a process of counting photons, where each photon is converted into an electric current.

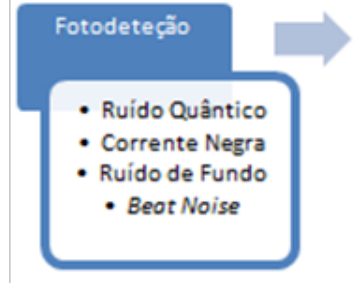

Figure 8 Blocks of the receiver and their noises.

The term of coherent detection, is used when you want to use

${ }^{2}$ This distribution represents the probability that an event occurs one at specified times in a time interval (space), when the occurrence rate is fixed. It

is given by the expression: $P(x)=\frac{\lambda^{x} * e^{-\lambda}}{x !}$. The parameter $\mathrm{x}$ is the random

variable, the number of occurrences of the event within a period of time, $\lambda$ is the occurrence of the event, the letter e is the constant of nepler approximately 2.71828 (constant). You can also set the parameters of the Poisson distribution, the average $(\lambda)$ and the standard deviation $(\sigma=\lambda)$.

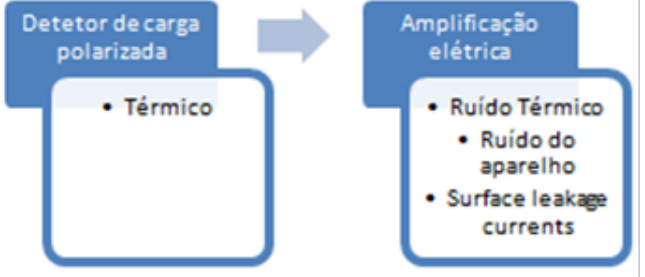

information about the phase and frequency of the signal. For this it is necessary that there should be a process prior to detection by the photodiode. The basic block for this processing is an optical oscillator site. The sign of this oscillator, represented by a laser diode, is "involved" in the receiver along with the received signal. The coherent detection can be divided into homodínica detection and face detection heterodínica. In summary, the receiver heterodínico, the received signal is applied to the signal of the oscillator lease. A homodínico receiver, the frequency of $\mathrm{LO}$ and its respective phase, are controlled 
in such a way that are equal to the phase and frequency of the received signal. The advantages of the coherent detection compared to direct detection are $^{2,6,11}$

a. Angle of modulation: the receiver is consistent can modulate both the frequency, as the phase;

b. Front end: Due to the limitations imposed by the quantum noise, the receiver of a coherent receiver can be easily readjusted to achieve to maximize the responsiveness and the bandwidth of the receiver. It is possible to increase the pace of transmission;

c. Selectivity of frequency;

d. Greater sensitivity;

The disadvantages of the coherent detection compared to direct detection are $2,3,6,11$

a. System complexity increases due to the LO;

b. Lasers: A spectral line of semiconductor lasers for standard is too large to be used with the reception is consistent.

\section{Sensibility}

Receiver sensitivity $P_{o p t}^{(\min )}$ is defined as the average power point minimum required at the input of the receiver for a given probability of error, (the probability of incorrect identification of the circuit decider, usually between $10^{-9}$ e $10^{-12}$ ), which leads to the quality factors between 6 to 7 . When you have multiple receivers, the most sensitive will be the one you can have the expected performance, with a lower power optical drive.

To determine the sensitivity on the receiver, it is necessary to consider the quality factor $(\mathrm{Q})$ in conjunction with the optical power mean low which will be needed to the receiver. Following the definition of the reason for revocation, the optical signal input, it relates the probability of the entrance there is a bit " 0 " with the likelihood of there is a bit "1". If the odds are equals, the average power optics to entry will be given by the expression

$\overline{p_{i}}=\frac{p_{i, 0}+p_{i, 1}}{2}$

The reason being, in this case, given by the expression

$$
r=\frac{p_{i, 0}}{p_{i, 1}}
$$

The average power optics to entry can be given by the equation

$$
\overline{p_{i}}==p_{i, 1} \cdot \frac{1+r}{2}=p_{i, 0} \cdot \frac{1+r}{2 r}
$$

To achieve relate the characteristics of the receiver will have to make reference to a set of formulas that allow us to understand the relationship between the quality factor and the sensitivity of the receiver. Through a set of expressions of probability conditional error from the bit " 0 " and the bit " 1 ", it is possible to remove the expression for the error probability of bit $p_{e}$ :

$$
P_{e}=\frac{1}{4}\left[\operatorname{erfc}\left(\frac{I_{D}-I_{0}}{\sigma_{0} \sqrt{2}}\right)+\operatorname{erfc}\left(\frac{I_{D}-I_{0}}{\sigma_{1} \sqrt{2}}\right)\right]
$$

The probability of error depends, as can be seen by the previous expression, the level of decision $I_{D}$. The choice at this level is done in order to reduce the maximum likelihood of error. For this choice takes as the basis of the expression

$$
\frac{I_{D}-I_{0}}{\sigma_{0}}=\frac{I_{1}-I_{D}}{\sigma_{1}}
$$

The expression for the level of decision has been amended by then:

$$
I_{D}=\frac{\sigma_{0} I_{1}+\sigma_{1} I_{0}}{\sigma_{0}+\sigma_{1}}
$$

All the expressions presented so far are related to the receptors PIN, as the power of noise on the receiver is essentially due to the noise from the circuit and not the quantum noise, $\sigma_{c}^{2} \gg \sigma_{q}^{2}$. Making the correct replacements, the error probability of bit will be given by the expression:

$$
P_{e}=\frac{1}{2} \cdot \operatorname{erfc}\left(\frac{Q}{\sqrt{2}}\right)=\frac{\exp \left(-\frac{Q^{2}}{2}\right)}{Q \cdot \sqrt{2 \pi}}
$$

With all the explanation was made, it is important to relate the input power with the quality factor. Thus, it follows the expression:

$$
Q=\frac{R_{\lambda}\left(P_{i, 1}-P_{i, 0}\right)}{2 \sqrt{\sigma_{c}^{2}}}
$$

Finally, results in the expression of receiver sensitivity, the type PIN, with dominant circuit:

$$
\overline{p_{i}}=\frac{1+r}{1-r} \cdot \frac{\left.Q \sqrt{S_{c}(f)} \sqrt{B_{e, n}}\right)}{R_{\lambda}}
$$

It is sometimes provided the value of the equivalent power of noise (NEP), which simplifies the expression above, resulting in the expression

$$
\overline{p_{i}}=\frac{\left.Q \sqrt{S_{c}(f)} \sqrt{B_{e, n}}\right)}{R_{\lambda}}
$$

In the case of recipients of APD type the reasoning is identical, and the approximations and similar results..$^{14,17}$

\section{Operation of optical receiver}

This optical receiver, low cost, is designed to operate at $10 \mathrm{Mbps}$, has as its main components a photodiode, a MOSFET two ports, one operational amplifier, among other components, such as: resistors, capacitors and transistors. The receiver has as initial constituent the photodiode. The photodiode used was the OPT101 the company Texas Instruments. This component receives the light beam emitted by laser, turning it into a electrical impulse. This current signal is converted into a voltage signal through a resistive feedback, which causes an amplification of transimpedance (TIA - transimpedance amplifier). It means that in parallel with the photodiode is used a resistance ( $R_{\text {tia }}$ ) and a low noise amplifier, which in conjunction with the MOSFET, convert the current signal to a voltage signal. Taking into account the noise introduced by the common mode, it is conceived a structure to somehow suppress this predicament, which implies careful in sizing the resistive component $\left(R_{t i a}\right)$. 
The photodetector and the MOSFET acquire a capacitance ruling, about $13 \mathrm{pF}(11 \mathrm{pF}+2 \mathrm{pF})$, in its operation. Through the conversion of a current signal to a voltage, there is a gain of transimpedance given by the expression:

$$
\mathrm{G}_{\mathrm{MOSFET}}=-\frac{R_{t i a}}{1+s R_{t i a} C_{o x}} \cdot g_{m} \cdot R_{D}
$$

In this equation, $g_{m}$ represents the transconductance inserted by the MOSFET, $C_{o x}$ indicates the capacitance of the cathode of the photodetector, and $R_{D}$ is the resistance associated to the filter becomes high, the resistance connected to the drain of the MOSFET.

The equation shows that there is an interference capacitance, which leads to losses caused by low pass filter, a response characteristic of a continuous signal analog (AC). In order to obtain the signal fully recovered after the reception in the photodetector, projecting the values of $R_{\text {tia }}$ and $R_{D}(100 \mathrm{k} \Omega$ and $560 \Omega$ ) according to the value of $g_{m}(27 \mathrm{mS})$, initially obtained with the input of $0 \mathrm{~V}$ and $4 \mathrm{~V}$ in the first and second door, respectively, of the MOSFET, according to a resistance of $2.2 \mathrm{M} \Omega$ and a resistive divider potential, performed by both resistance of $180 \mathrm{k} \Omega$ and $82 \mathrm{k} \Omega$ fed by a continuous voltage of $12 \mathrm{~V}$.

Subsequently, the operational amplifier, assembled for the purpose of performing the functions of differentiator and amplifier, is on a specific capacitance and dependent on the pace tighten used on connection. The last part of the circuit is the recovery of the signal. It is called an amplifier that will limit the signal to produce so recover in full the signal. This part is composed by transistors of type n-p-n $2 \mathrm{~N} 3904$, which form a pair of long tail. The resistance of $820 \Omega$ is used to define the forward bias current. The $75 \Omega$ represent the resistivity coaxial cable and allows you to maximize the power transmitted by the interface of the receiver. Added a makeup of current composed of two transistors $2 \mathrm{~N} 1410$, as well as was essential to move the coax cable to the exit point of the circuit, in parallel with the capacitor which is located at the end, so as to be able to record data more conclusive. It is then a block diagram, which represents the various stages through which the signal passes to the receiver optics (Figure 9).
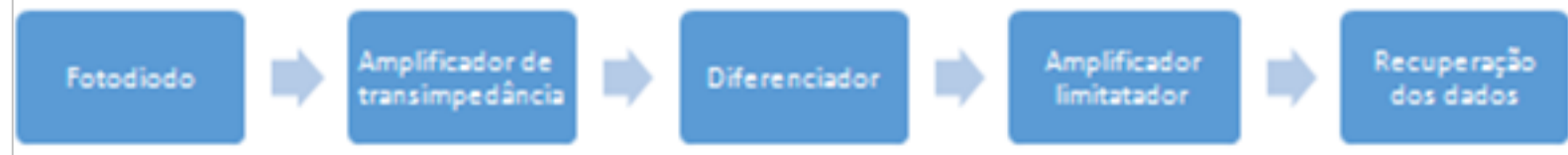

Figure 9 Diagram of the optical receiver.

In Figure 9 the components that belong to and constitute the building blocks are, at the beginning, the monolithic photodetector OPT101, from Texas Instruments, common source and amplifier of transimpedance. The signal is received at the goal of abstraction of the receiver and then is amplified. In the second block, is this MOSFET BF998 silicon and N-channel of two ports, from NXP Semiconductors, as well as the components resistance values that allow the transformation of current signals to voltage. The key differentiator is essentially the Operational Amplifier TL 592b, video amplifier, differential from Texas Instruments. The limitation and recovery of the signal is constituted by transistors and their resistance.

\section{Components of the optical receiver}

\section{Photodetetor}

Its operation depends on the incidence of the optical signal with a certain wave length. Still in the same figure, you can observe all the links of the respective component as well as the scheme in which he falls. In this figure, the receiver supply voltage $\left(\mathrm{V}_{\mathrm{cc}}\right)$ is $12 \mathrm{~V}$, and the input signal $\left(\mathrm{V}_{\text {in }}\right)$ is a signal with amplitude of $5 \mathrm{~V}$. The frequency of testing ranged from $1 \mathrm{kHz}$ to $1 \mathrm{MHz}$ interspersed with an order of magnitude between values. The supply voltage of the photodiode $(\mathrm{V}+)$ used was $6 \mathrm{~V}$.

\section{MOSFET two ports}

The fact that it is a MOSFET two ports, allows us to affirm that both have an effect on the current signal that is located between the source and drain. How is all of the configuration, and even taking into account the capacitors that are not present in this analysis, since it is an analysis DC, indicates that this configuration serves to amplify the signal. With the use in series two FETs (Field Effect Transistor-Field Effect Transistor) into one component, it is possible to modeling of a MOSFET, with two floors of amplification in cascade.

This layout allows overcoming the effect of Miller, which affects the capacitance between the input stage and the output. Not forgetting that this effect may be related to impedance, the more harmful to the signal are the capacitance of the components. The effect of Miller is exceeded by the use of an amplifier in cascade, as has already been mentioned, polarizing the second door of MOSFET. The third pin, with a value of constant potential, reduces the loss of profit caused by the effect of Miller. Thus, the effects of capacitive coupling between the input and the output are virtually wiped out. To study the MOSFET, it is ignored the mode of operation in court, with special attention being paid to other modes of operation, the triode and saturation. Thus, the following table allows you to summarize the areas of operation of the MOSFET amp for two doors (Table 2).

Table 2 Operation modes of a MOSFET dual gate

\begin{tabular}{llll}
\hline Region & $\mathbf{I}^{\circ}$ FET & $\mathbf{2}^{\mathbf{o}}$ FET & $\mathbf{M}$ \\
\hline $\mathbf{I}^{\mathrm{a}}$ & Triode & Triode & -- \\
$2^{\mathrm{a}}$ & Triode & Saturation & Gain reduction \\
$3^{\mathrm{a}}$ & Saturation & Triode & -- \\
$4^{\mathrm{a}}$ & Saturation & Saturation & Total gain
\end{tabular}

For a FET works in the region of triode have to check two conditions, i.e., the tension between the source and the door must be higher than the threshold voltage ( $V_{t h}$ threshold), and the tension between the drain and source $\left(V_{D S}\right)$ must be less than the result of the subtraction between the voltage-source $\left(V_{G S}\right)$ and the threshold, i.e., $V_{G S}>V_{t h} V_{D S}>V_{G S}-V_{t h}$. When these conditions occur, there is current flow between the Drain and the Source. The MOSFET operates as a resistance and is controlled by the voltage on your doorstep. The chain of the drain $\left(I_{D}\right)$ to the source is given by the expression:

$$
I_{D}=\frac{\mu_{n} C_{o x}}{2} \frac{W}{L}\left(2\left(V_{G S}-V_{t h}\right) V_{D S}-V_{D S}^{2}\right)
$$

The expression $\mu_{n} C_{o x}$ is, for the most part, replaced by parameter $k_{n}^{\prime}$, named as a parameter for transconductance. 
Even in this mode of operation, there are two distinct zones: one in which the function is approximately linear when $V_{D S} \ll V_{G S}$ and other sub-linear when $V_{D S} \approx V_{G S}$.

If you want to use a FET as amplifier, is not in triode that operates, even if there is a linear zone in this mode of operation, but in the region of saturation. For a FET works in the region of saturation, have to also check two conditions, that is, the voltage-source must be higher than the threshold voltage of the FET as well, the tension drain-to-source must be higher than the subtraction between the voltage-source and the voltage threshold, i.e., $V_{G S}>V_{t h}$ e $V_{D S}>V_{G S}-V_{t h}$. It is created in the same way a channel that allows the passage of a current flow between the drain and the source, but as the voltage drain is higher than the voltage at the door, a part of this channel is switched off. This region formed is called the pinch-off, i.e., there is a "strangling" of the channel, getting narrower for current flow. Thus, it is possible a relative independence of drain-current in relation to the voltage from drain. This current flows to be controlled by the tension in the gate, giving rise to the expression:

$$
I_{D}=\frac{\mu_{n} C_{o x}}{2} \frac{W}{L}\left(V_{G S}-V_{t h}\right)^{2}
$$

For a MOSFET polarized well in the region of saturation with a particular current $I_{D}$ and a voltage $I_{D}$, he gain of transconductance ( $\left.g_{m}\right)$ is given by the expression:

$$
g_{m}=\frac{2 I_{D}}{\left(V_{G S}-V_{t h}\right)}
$$

It is also important to mention the region of cut that happens when $V_{G S}<V_{t h}$. In this case there is virtually current between drain and source. It is affirmed once more the use of MOSFET, so as to implement an amplifier, managing to make the voltage in the second door $V_{G_{2} S}$, with a DC source along with a signal $\mathrm{AC} V_{G_{1} S}$. Thus, the gain of the amplifier circuit ( $G_{a}$ ) shall be given by the expression:

$$
G_{a}=g_{m} \cdot R_{D}
$$
$R_{D}$.

The resistance which is connected to the drain is represented by

\section{Differential amplifier}

The layout of the AMPOP used is shown in Figure 10, along with an explanation of the functions that are present in your schematic.

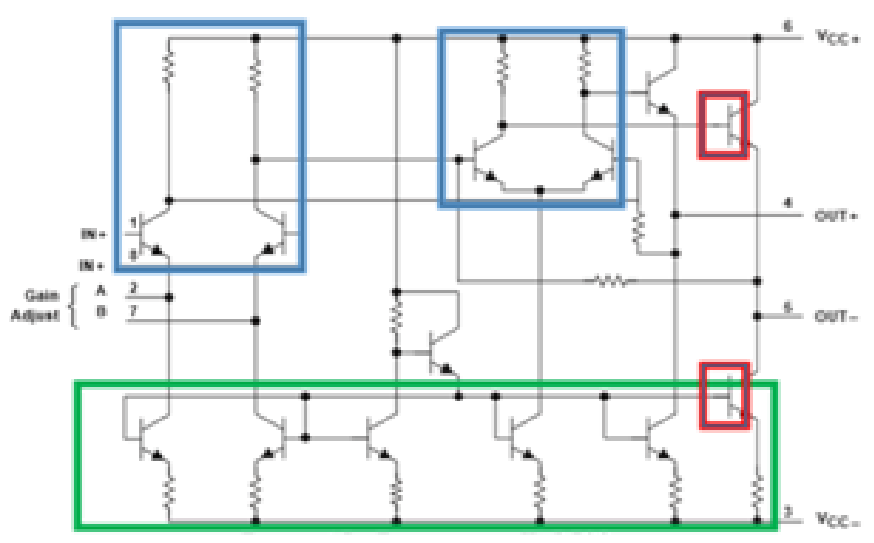

Figura 10 Outline of the TLB592b.
Through the analysis of the schematic of differentiating, it should be noted the existence of a differential pair (blue rectangle) and polarization with makeup of current (green rectangle) (Figure 11).

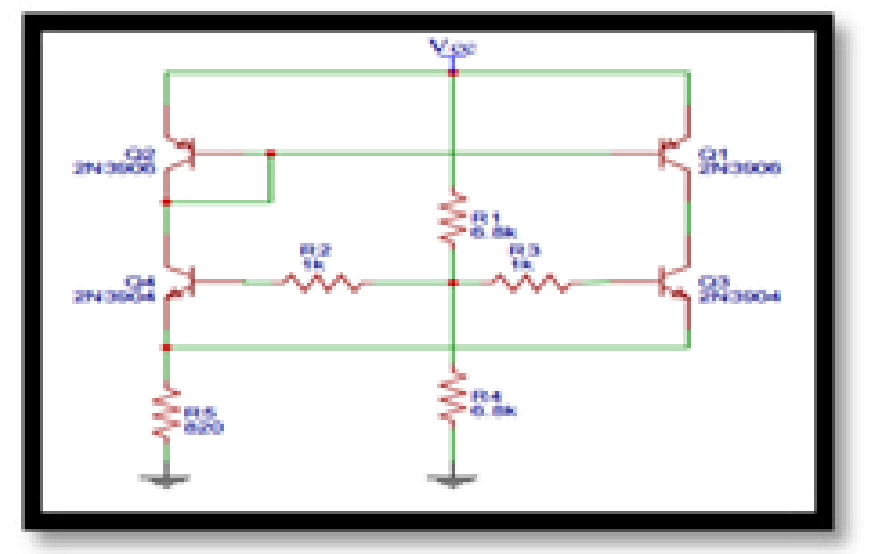

Figure II Recovery of the final signal.

\section{Recovery of signal}

A differential pair has as functions, the differences, serve as operational amplifiers and allow the conversion of differential signals in signals not differentiators. In summary form, to make real a differential pair with active load with output in one of the collectors (Q3), instead of being used two sources of independent power that would be linked to the transistors Q3 and Q4, it resorts to a repeater. ${ }^{18-21}$

Some of the expressions that allow studying the transistors, to be able to determine which modes of operation operate are as follows:

$$
\begin{aligned}
& v_{B C}+v_{E B}-v_{E C}=0 \\
& v_{C B}+v_{B E}-v_{C E}=0 \\
& i_{B}+i_{C}=i_{E} \\
& i_{C_{1}}=i_{C_{2}}=\frac{I_{E E}}{1+e^{\frac{V_{D}}{V_{t}}}} \\
& i_{C}=i_{b} * \beta \\
& i_{C}=\frac{\beta}{1+\beta} * i_{e} \\
& i_{c}=I_{S} * e^{\frac{V_{B E}}{V_{t}}}
\end{aligned}
$$

As for the regions of operation in which the transistor can work, are listed in Table 3:

Table 3 Operation modes of a bipolar junction transistor

\begin{tabular}{lll}
$\begin{array}{l}\text { Mode of } \\
\text { operation }\end{array}$ & Junction BE & Junction BC \\
\hline cut & Polarized inversely & Polarized inverselyinversamente \\
Active & Polarized directly & Polarized inversely \\
Saturation & Polarized directly & Polarized directly \\
\hline
\end{tabular}

In Table 3, when referring to the forward bias, it is understood that the diode is ON, i.e., $V_{D}=V_{O N}$ 


\section{Tests and printed board}

The purpose of this dissertation ended with the projection and construction of a printed plate. The modeling of the same was done in the program EasyEDA and components are organized according to the layout of the optical receiver. With regard to the printed plate, all components are easily identifiable. There is also the fact that the board is designed to operate independently of other devices due to the sources of tension. The two colors used, the red and blue, indicating the two floors of the plate. Requires two floors to get log all connections (Figure 12, Figure 13).

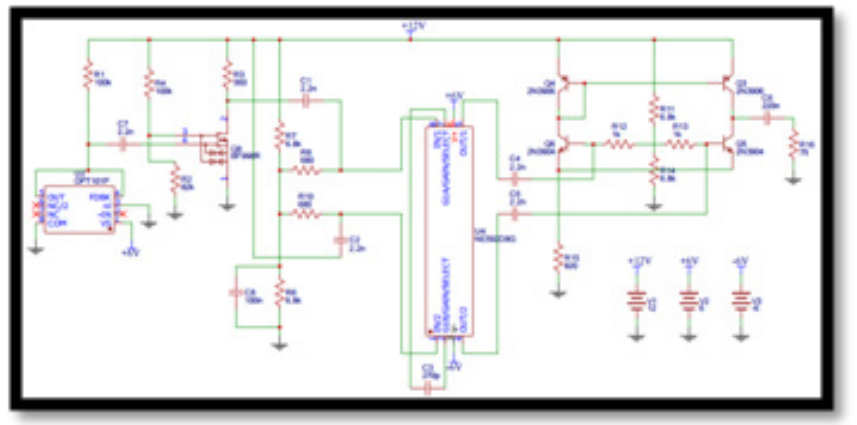

Figure 12 Optical receiver on breadboard.

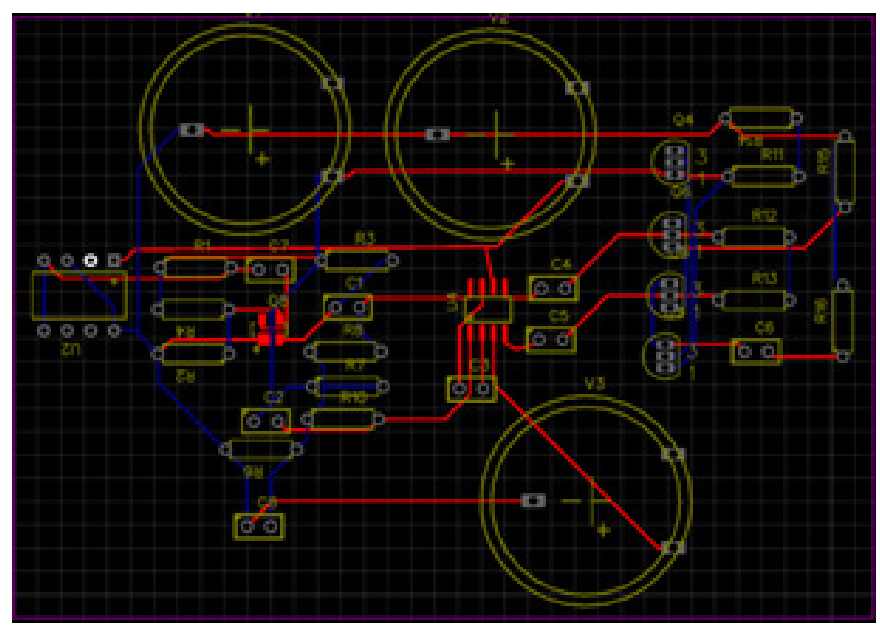

Figure 13 Optical receiver in a printed board.

The tests conducted were performed at $100 \mathrm{kHz}$ and the $1 \mathrm{Mhz}$. The received signal is $5 \mathrm{~V}$ and demonstrates the value of the output signal of the optical receiver. With the increase of the frequency, the results are increasingly positive (Figure 14, Figure 15).

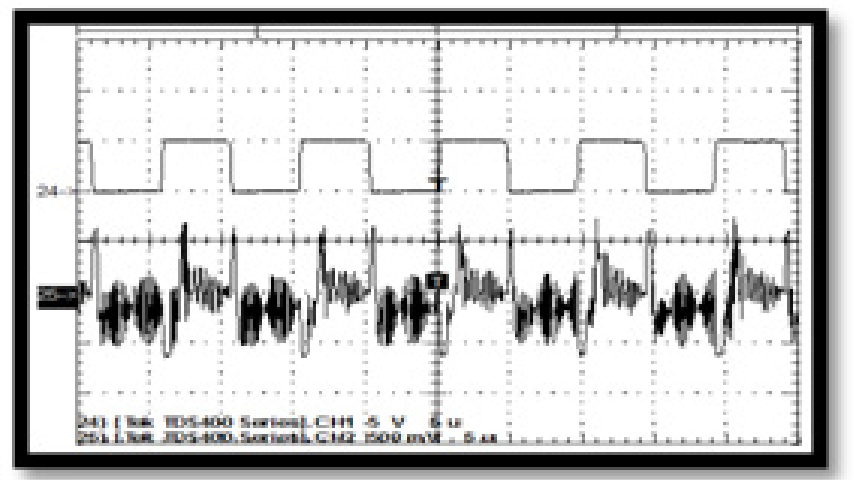

Figure I 4 An output signal from the receiver to $100 \mathrm{kHz}$.

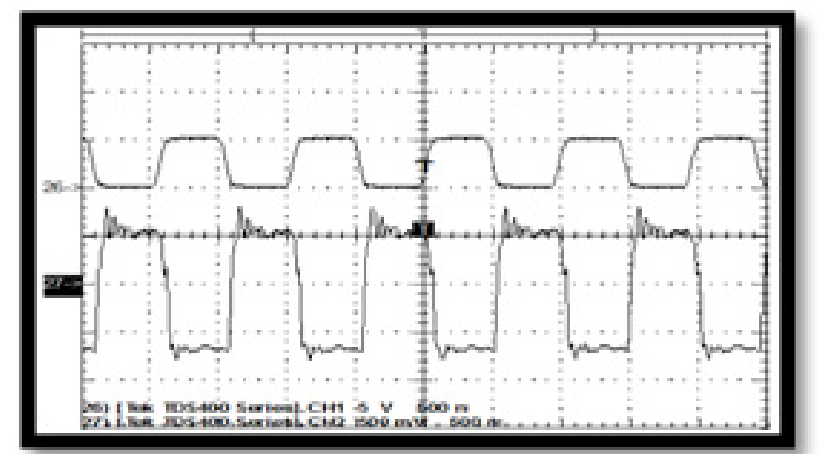

Figure 15 The output signal from the receiver to $1 \mathrm{MHz}$.

\section{Conclusion}

Considering the fact that the optical communications are present, increasingly, in our daily lives, the environment and the environment military, this dissertation had the objective of drawing and construction of a receiver optical, in printed plate, which fulfilled all the goals defined initially. The study in a first stage made it possible to understand the communication system that involves the optical receiver. Also allowed to characterize the components required for the receiver optics perform all functions, by blocks, necessary for the reception and recovery of the signal sent by a transmitter. It was found that the more complex is the modulation more secure for communication. Consequently, with the increased security, increases the complexity and difficulty of implementing. It was concluded that the spatial environment is an environment of increased wear to the electronic systems and in this way we use materials more robust and resistant, as is the case of raw military. In contrast, in an environment of free space, it is advantageous for the loss of the signal because the attenuation that would be made by the earth's atmosphere and is not made by the space environment. It was referred to the PAT, which allows the sender and receiver are situated, connect, and then exchange information. The results were evidence to assert that the receiver optical can perform required functions. It is thus the objectives outlined earlier. The culmination of the paper passed by producing a printed plate. This board is the implementation of the entire circuit of optical receiver, with the inclusion of the sources of tension. In this way, allows operating with the optical receiver anywhere, without any dependencies with other materials.

\section{Acknowledgments}

None.

\section{Conflicts of interest}

Author declares that there are no conflicts.

\section{References}

1. Florenzano TG. Os Satélites e Suas Aplicações. 1st ed. Brazil: SindCT; 2008. $50 \mathrm{p}$.

2. Herzog FT. An optical phase locked loop for coherent space communications. ETH. 2006.

3. INPE. Quais os tipos de satélite artificiais que existem? 2016.

4. Pinto AN. Análise e Optimização de Sistemas de Comunicação Ópticos Baseados em Solitões. 1999. 324 p.

5. Bindushree AP, Nataraju AB, Vijesh TV, et al. Design And Simulation Of QPSK Modulator For Optic Inter Satellite Communication. International journal of scientific \& technology research. 2014;3(8):402-408. 
6. Rasmussen J, Hoshida T, Nakashima H. Digital Coherent Receiver Technology for 100-Gbps Optical Transport Systems. FUJITSU Sci Tech J. 2009;46(1):476-483.

7. Karppinen M, Tanskanen A, Ollila J, et al. Fiber-optic transceivers for high-speed intra-satellite links. Avionics. Fiber-Optics and Photonics Technology Conference. 2012;3:34-35.

8. Alluru SR. A system design of an optical wireless communication system for cubesats. $2010.78 \mathrm{p}$.

9. Redes de comunicação. Modulação. 2012.

10. Cartaxo. Transmissão por Fibra Óptica. Lisboa: Instituto Superior Técnico. 2005.

11. Vítor M, Coelho M. Simulador de Receptor Óptico Digital de Modulação de Intensidade e Detecção Directa. 2009.

12. Hanzra TS, Singh G. Performance of Free Space Optical Communication System with BPSK and QPSK Modulation. Electronics and Communication Engineering. 2012;1(3):38-43.
13. Malburg, M. Trabalho final de redes I. Universidade Federal do Rio de Janeiro. Departamento de engenharia eletrônica. 2004.

14. Semenova Y. Optical Communications Systems Optical Receivers. Dublin: Dublin Institute of Technology; 2003.

15. Fernando X. Optical Receivers-Theory and Operation. 2016.

16. Rebola JL. Photodetectors. Lisboa: ISCTE; 2011

17. Oscarsson J. Simulation of Optical Communication for Formation Flying Spacecraft. UPPSALA; 2008. 109 p.

18. Hemmati H, June F. Laser Communications : From Terrestrial Broadband to Deep-Space. Transparent Optical Networks (ICTON). 2014

19. Riebeek H. Catalog of Earth Satellite Orbits. 2009

20. Kharraz O, Forsyth D. Performance comparisons between PIN and APD photodetectors for use in optical communication systems. International Journal for Light and Electron Optics. 2013;124(13):1493-1498.

21. Agrawal GP. Fiber-Optic Communications Systems. 3rd ed. Rochester: A john wiley \& sons; 2002. 561 p. 